\title{
Voicing Detection in Noisy Speech Signal
}

\author{
Aïcha Bouzid ${ }^{1}$ and Noureddine Ellouze ${ }^{2}$ \\ ${ }^{1}$ Institut Supérieur d'Electronique et de Communication de Sfax, Route Menzel Chaker \\ $\mathrm{Km}$ 0.5, B. P. 868, 3018 Sfax, Tunisia \\ ${ }^{2}$ Ecole Nationale d'Ingénieurs de Tunis, BP. 37, Le Belvédère 1002 Tunis, Tunisia \\ bouzidacha@yahoo.fr, N.Ellouzedenit.rnu.tn
}

\begin{abstract}
An algorithm for voicing detection in noisy speech signal is proposed. This algorithm is based on the product of wavelet transforms at some scales called multi-scale product. The multi-scale product has the ability to reinforce the edge in the signal while suppressing additive noise. Motivated by the fact that unvoiced sounds are, in most important speech production models, considered as filtered noise, we apply the multi-scale product on speech signal for detection of voiced segments. In fact, the multi-scale product anneals the signal frames corresponding to unvoiced sounds and frames of silence, while it conserves speech periodicity for voiced frames.
\end{abstract}

Keywords: Wavelet transform, multi-scale product, voicing decision, speech signal.

\section{Introduction}

Pre-processing of speech signal is very crucial in the applications where silence or background noise is completely undesirable. Applications like speech and speaker recognition [1] needs efficient feature extraction techniques from speech signal where most of the voiced part contains speech or speaker specific attributes. Silence removal is a well known technique adopted for many years for this and also for dimensionality reduction in speech that facilitates the system to be computationally more efficient. This type of classification of speech into voiced or silence/unvoiced sounds [2] finds other applications mainly in fundamental frequency estimation, formant extraction or syllable marking and so on.

There are several ways of classifying events in speech. It is accepted convention to use a three state representation in which states are (i) silence where no speech is produced; (ii) unvoiced sound, in which the vocal cords [3] are not vibrating, so the resulting speech waveform is random in nature and (iii) voiced sound, in which the vocal cords are tensed and therefore vibrate periodically when air flows from the lungs, so the resulting waveform is quasi-periodic [4]. It should be clear that the segmentation of the waveform into well defined regions of silence, unvoiced, signals is not exact; it is often difficult to distinguish a weak, unvoiced sound (like /f/ or /th/) from silence, or weak voiced sound (like $/ \mathrm{v} /$ or $/ \mathrm{m} /$ ) from unvoiced sounds or even silence.

The speech classification task has been studied in many articles by a variety of approaches since 1980's. Basically the classification is done by relying on different 
types of feature vectors which are extracted from the input speech frames. These features can be derived by three approaches:

The first approach works in the time domain and uses statistical measurements. The common features are zero crossing rate, relative energy level, autocorrelation coefficients, etc. [5], [6], [7]. This approach only achieves good accuracy if using a large number of parameters.

The second approach works in the frequency domain. Frequently used features are the spectrum [8], optimal filters [9], Mel Frequency Cepstral Coefficients (MFCC) [10]. The results show that MFCCs give high performance.

The third approach combines both time and frequency domains, using the Short Time Fourier Transform (STFT) [11] or Wavelet Transform (WT) [12].

In this paper, we propose a time-scale approach using multi-scale product of wavelet transform for the detection of voicing frames in speech. The paper is organized as follows. The second section puts forward the time-scale analysis of signals. The third section concerns the edge detection of noisy signal by the multiplication of wavelet transform coefficients. Section 4 presents the proposed method for voicing detection. Section 5 concludes this work.

\section{Wavelet Transform Modulus Maxima}

Wavelet Transform is a linear powerful time-scale representation of signals, a real wavelet $\psi(\mathrm{t})$ which has the property of zero mean is used for this representation.

$$
\int \psi(\mathrm{t}) \mathrm{dt}=0 \text {. }
$$

The wavelet is delayed by a time factor $u$ and scaled by a scale factor $s$, the basic expression of wavelet transform of the signal $f(t)$ is

$$
\mathrm{Wf}(\mathrm{u}, \mathrm{s})=\int \mathrm{f}(\mathrm{t}) \frac{1}{\sqrt{\mathrm{s}}} \psi\left(\frac{\mathrm{t}-\mathrm{u}}{\mathrm{s}}\right) \mathrm{dt} \text {. }
$$

This expression of correlation can be written as a convolution product with the time reverse function $\bar{\psi}_{s}(u)$

$$
\bar{\psi}_{\mathrm{s}}(\mathrm{u})=\frac{1}{\sqrt{\mathrm{s}}} \psi\left(\frac{-\mathrm{u}}{\mathrm{s}}\right) .
$$

The convolution expression is:

$$
\mathrm{Wf}(\mathrm{u}, \mathrm{s})=\left(\mathrm{f} * \bar{\psi}_{\mathrm{s}}\right)(\mathrm{u}) .
$$

This can be interpreted as a linear filtering with the impulse response $\bar{\psi}_{s}(u)$. This equation gives the ability of a filter bank design. A wavelet orthogonal basis can be constructed for discrete parameters $s=2^{\mathrm{j}}$ and $\mathrm{u}=\mathrm{i} 2^{\mathrm{j}}$, named dyadic wavelet transform.

The wavelet transform can be used for different applications. According to Mallat, the wavelet transform, has shown excellent capacities for the detection of signal singularities. When the wavelet function has specific selected properties, wavelet 
transform acts as a differential operator. The number of wavelet vanishing moments gives the order of the differentiation. A wavelet $\psi(\mathrm{t})$ is said to have $\mathrm{n}$ vanishing moments, when for all positive integer $\mathrm{k}<\mathrm{n}$, it satisfies the following equation [13]

$$
\int_{-\infty}^{+\infty} \mathrm{t}^{\mathrm{k}} \psi(\mathrm{t}) \mathrm{dt}=0 \quad 0<\mathrm{k} \leq \mathrm{n} .
$$

A wavelet $\psi(\mathrm{t})$ with a fast decay has $\mathrm{n}$ vanishing moments if a function $\theta$ has a fast decay such that

$$
\psi(\mathrm{t})=(-1) \frac{\mathrm{n}}{\mathrm{d}^{\mathrm{n}} \theta(\mathrm{t})} \mathrm{dt}^{\mathrm{n}} .
$$

This function is called a smoothing function. When the wavelet has $\mathrm{n}$ vanishing moments the equation 4 can be written as

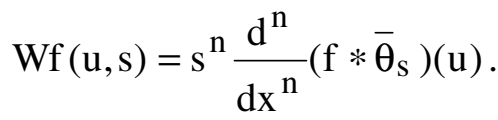

Which is a differential operator of order $n$ of the function $f(t)$ smoothed by the function $\bar{\theta}_{\mathrm{s}}[14]$

$$
\bar{\theta}_{\mathrm{s}}=\frac{1}{\sqrt{\mathrm{s}}} \theta\left(\frac{-\mathrm{t}}{\mathrm{s}}\right) .
$$

Modulus maxima of wavelet coefficients describe the instants such that $|\mathrm{Wf}(\mathrm{u}, \mathrm{s})|$ is locally maximum.

$$
\frac{\partial \mathrm{Wf}(\mathrm{u}, \mathrm{s})}{\partial \mathrm{u}}=0 \text {. }
$$

This equation defines in the time-scale plane, lines corresponding to discontinuities at maximum amplitude of Wf $(\mathrm{u}, \mathrm{s})$ [13].
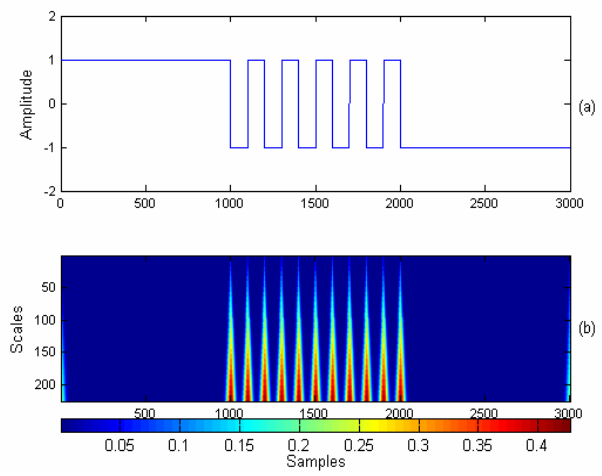

Fig. 1. (a) Square signal and (b) its wavelet transforms at scales ranging from $1 / 4$ to 2 
When the wavelet has one vanishing moment, modulus maxima, are the maxima of the first derivative of the smoothed signal at discontinuities [14]. If the wavelet transform has no modulus maxima at fine scales then the function is locally regular. Hence singularities are detected by finding abscissa, where the wavelet modulus maxima converge at fine scales.

Figure 1 gives an example of wavelet modulus maxima amplitude of a square signal. The wavelet used is the quadratic spline wavelet having obviously one vanishing moment.

\section{Multi-scale Product for Noisy Signal Edge Detection}

Working before the advent of the wavelet framework, Rosenfeld et al suggested forming multi-scale point-wise products [15]. This is intended to enhance multi-scale peaks due to edges, while suppressing noise, by exploiting the multi-scale correlation due to the presence of the desired signal. The multi-scale product (MP) of the dyadic wavelet transform is given by [16], [17]

$$
\mathrm{p}(\mathrm{u})=\prod_{\mathrm{j}=\mathrm{J}_{0}}^{\mathrm{J}} \mathrm{Wf}\left(\mathrm{u}, 2^{\mathrm{j}}\right)
$$

In equation $10, W f\left(u, 2^{j}\right)$ represents the wavelet transform at scale $\mathrm{s}=2^{\mathrm{j}}$, and the product is operated for scales $2^{\mathrm{J}}, 2^{\mathrm{J}-1} \ldots 2^{\mathrm{J} 0}$. The expression of equation 10 is distinctly a non linear function. The product $\mathrm{p}$ reveals peaks at signal edges, and has relatively small values elsewhere. Singularities produce peaks along scale in wavelet transform, these peaks are reinforced by the product $\mathrm{p}(\mathrm{n})$. Although particular smoothing levels may not be optimal, the non linear combination tends to reinforce the peaks while suppressing spurious peaks. This is proved by figures 2 and 3 .

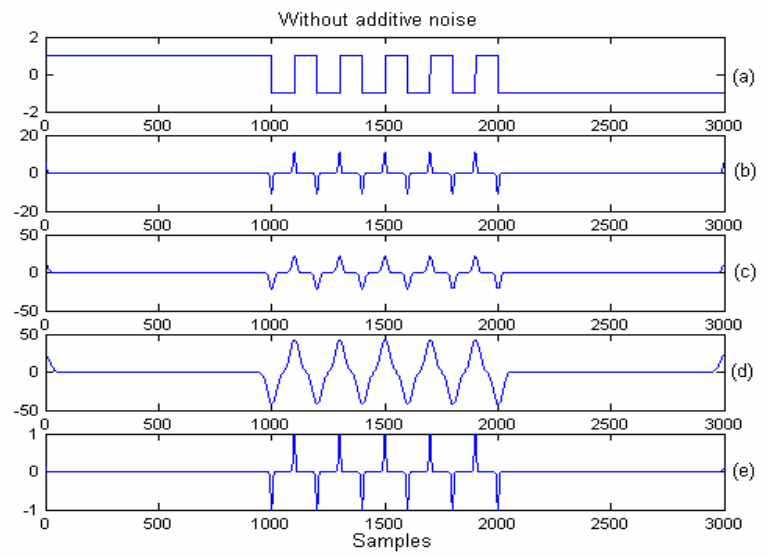

Fig. 2. (a) Square signal, (b) WT at scale $s=1 / 2$, (c) WT at scale $s=1$, (d) WT at scale $s=2$ and (e) the MP 
As illustrative example, figure 2 represents the wavelet transform (WT) of a square signal at the selected scales $(1 / 2,1$ and 2$)$ operated with the quadratic spline wavelet, and their products. For this case, the wavelet transform at the lowest scale and the MP permit a better edge localisation.

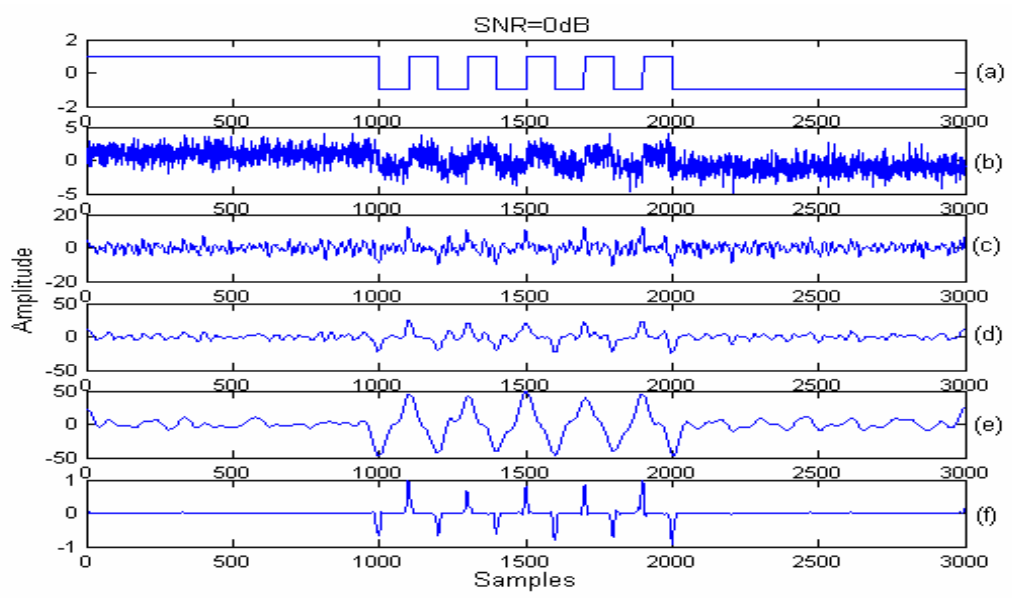

Fig. 3. (a) Representation of the square signal, (b) the signal with additive noise $(\mathrm{SNR}=0 \mathrm{~dB})$, (c) WT at scale $s=1 / 2$, (d) WT at scale $s=1$, (e) WT at scale $s=2$ and (f) the MP

Figure 3 illustrates the effect of the wavelet transform and the MP on a signal corrupted with a Gaussian white noise and having an SNR=0dB. The effect of the smoothing due to the scale increasing reduces dramatically the noise power. The noise is completely eradicated by the multi-scale product.

\section{Voicing Decision Based on Multi-scale Product}

The objective of this section is to illustrate the discontinuity detection capacity of the multi-scale product of speech in presence of powerful additive Gaussian noise. We will show that the multi-scale product reduces additive noise and the intrinsic noise, in other hand it enhances voiced parts of speech and this can be used to operate a voicing classification.

In [18], the multi-scale product is applied on voiced sounds in order to detect speech edges. The cross scale product depicts two types of peaks; minima corresponding to glottal closure instants (GCIs) and maxima related to glottal opening instants (GOIs). In this section, we apply the multi-scale product to a clean and noisy speech signal for voicing detection. The wavelet transforms are calculated at the following successive dyadic scales $1 / 2,1,2$ corresponding to $j$ values of $-1,0$ and 1 . To show the efficiency of the proposed method, we illustrate in fig. 4(a) the speech signal composed by a silence, voiced and unvoiced sounds corresponding to the segment /and the sun/ extracted from the sentence /the north wind and the sun were disputing which was the stronger/ and uttered by the female speaker f1 of the Keele University database [19]. 


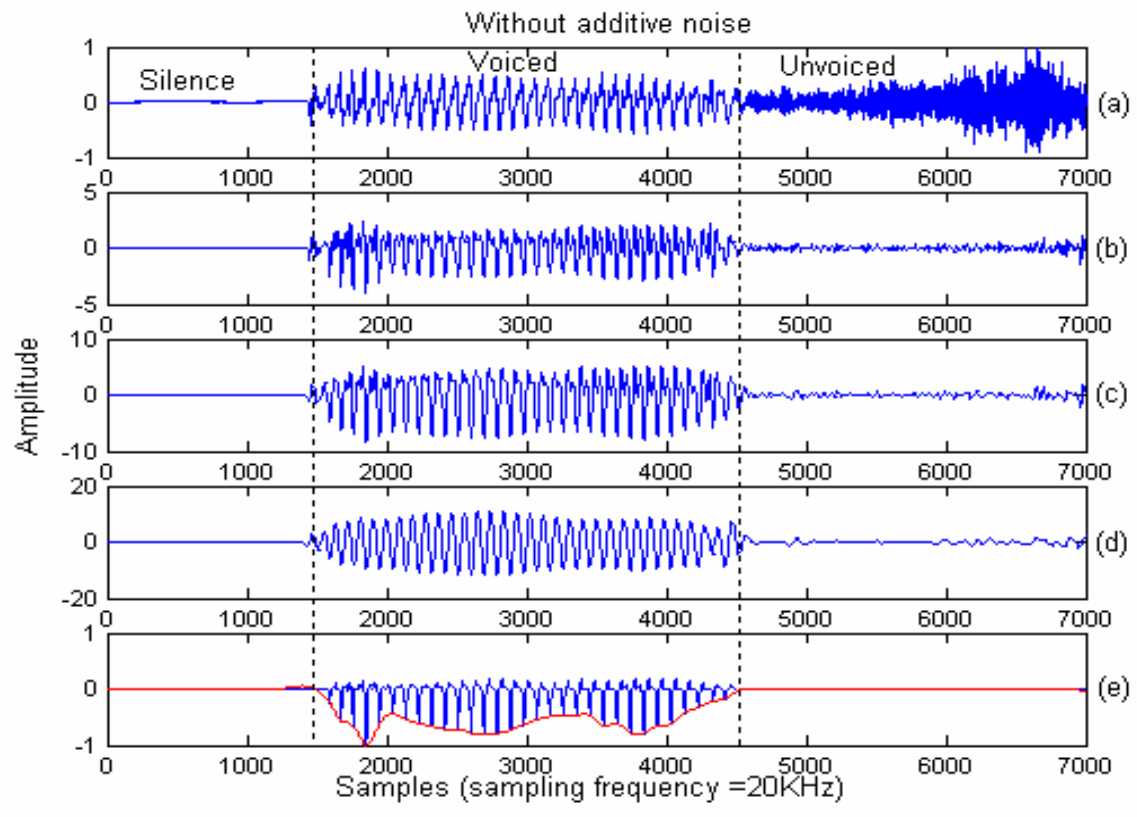

Fig. 4. Voicing decision, (a) speech signal, (b) WT at scale $s=1 / 2$, (c) WT at scale $s=1$, (d) WT at scale $s=2$ and (e) the product of the three scales giving the detection of the voiced sound

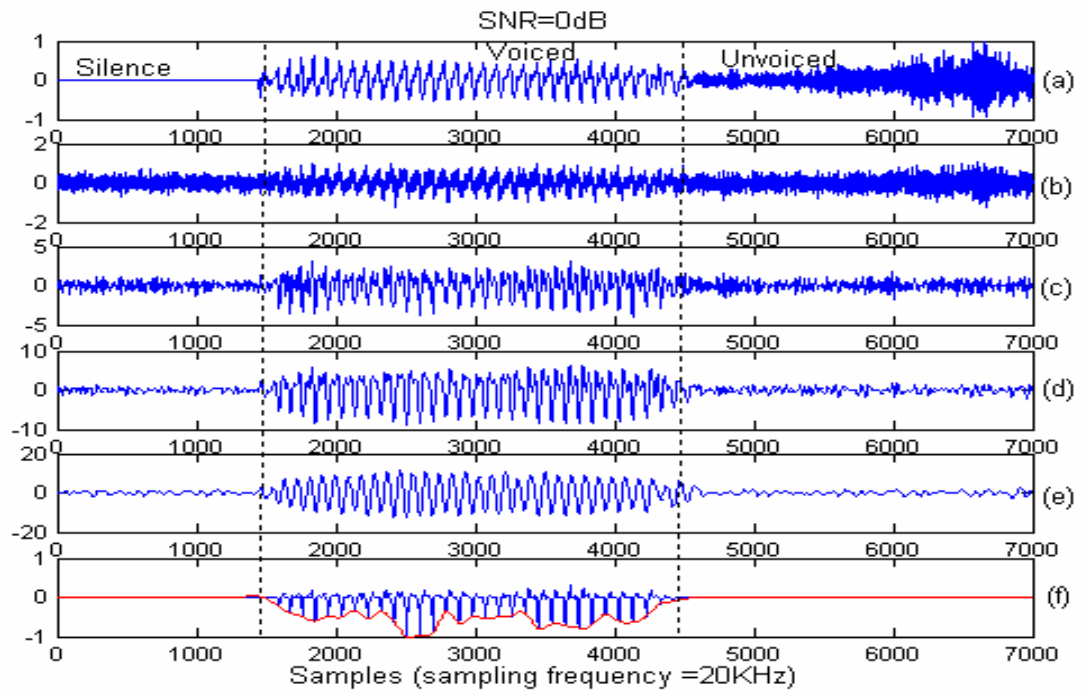

Fig. 5. Voicing decision, (a) speech, (b) speech+ white Gaussian noise $\mathrm{SNR}=0 \mathrm{~dB}$, (c) WT at scale $s=1 / 2$, (d) WT at scale $s=1$, (e) WT at scale $s=2$, (f) MP 
When the scale increases, the unvoiced sounds tend to be annealed by the wavelet transform. The multi-scale product eradicates completely the unvoiced sounds. In fact, in the signal of fig. 4(f), we can only visualize the voiced sound with a periodic structure. This behaviour allows us to make a classification of voiced / (unvoiced sounds or silence). To be able to make a decision, we construct the envelope of minima given by the multi-scale product. This envelope is non null for the voiced sounds. So decision on voicing is made when the corresponding envelope is not annealed.

This approach is also applied to a speech signal corrupted with a Gaussian noise. Figure 5 depicts the speech signal (fig. 5(a)) added to a Gaussian noise with an SNR equal to $0 \mathrm{~dB}$ (fig.5 (b)). Its WT at the following scales $1 / 2,1$ and 2 are given in fig. 5(c), fig. 5(d) and fig. 5(d). It is shown that at the finest scale, the wavelet coefficients are almost dominated by noise. At the second and third scales, the noise diluted rapidly. It can also be seen that at small scales the positions of the step edges are better localized. But some noise may be falsely considered as edge and the voiced classification can't be operated. At the large scales, the SNR is improved and edges can be detected more correctly but with the decreasing of the accuracy of the edge location.

We can clearly see the effect of the multi-scale product in eliminating the noise and the unvoiced sounds fig. 5(f). The MPM has the same behaviour regarding the noise and the unvoiced sounds.

\section{Conclusion}

In this work, we have described a new method for voicing decision in the case of noisy speech signal. The proposed approach is based on making the product of the speech signal wavelet transforms at three dyadic scales. This product has an appropriate behaviour regarding the voicing state. The multi-scale product reveals annealed values for silence and unvoiced sounds and has a periodic structure corresponding to voiced sounds. In fact the noise effect is eradicated thanks to the large scale multiplication that reduces the spurious peaks due to noise. This property allows to make a good classification of voiced / (unvoiced or silent), when a noise is added to the speech signal.

\section{References}

1. Campbell Jr., J.P.: Speaker Recognition: A Tutorial. Proceedings of the IEEE 85(9), 14371462 (1997)

2. Martin, A., Charlet, D., Mauuary, L.: Robust Speech/ Non-speech Detection Using LDA Applied to MFCC. ICASSP, vol. 1, pp. 237-240 (2001)

3. Ishizaka, K., Flanagan, J.L.: Synthesis of voiced Sounds from a Two-mass Model of the Vocal Chords. Bell System Technical J. 50(6), 1233-1268 (1972)

4. Atal, B., Rabiner, L.: A Pattern Recognition Approach to Voiced-unvoiced-silence Classification with Applications to Speech Recognition. IEEE Trans. On Signal Processing 24(3), 201-212 (1976)

5. Kedem, B.: Spectral Analysis and Discrimination by Zero-crossings. In: Proc. IEEE, vol. 74, pp. 1477-1493 (1986) 
6. Childers, D.G., Hahn, M., Larar, J.N.: Silence and Voiced/Unvoiced/Mixed Excitation Classification of Speech. IEEE Trans. On Acoust., Speech, Signal Process 37(11), 1771-1774 (1989)

7. Liao, L., Gregory, M.: Algorithms for Speech Classification. 5th ISSPA Brisbane, 623-627 (1999)

8. Zahorian, S.A., Silsbee, P., Wang, X.: Phone Classification with Segmental Features and a Binary-pair Partitioned Neural Network Classifier. In: ICASSP, pp. 1011-1014 (1997)

9. Niyogi, P., Sondhi, M.M.: Dectecting Stop Consonant in Continuous Speech. J. Acoust. Soc. Am. 111, 1063-1076 (2002)

10. Xiong, Z., Huang, T.: Boosting Speech/non-speech Classification Using Averaged Melfrequency Cepstrum. In: IEEE Pacific-Rim Conf. on Multimedia (2002)

11. Yang, H., Vuuren, S.V., Hermansky, H.: Relevancy of Time-frequency Features for Phonetic Classification Measured by Mutual Information. In: ICASSP, vol. 1, pp. 225-228 (1999)

12. Lachiri, Z., Ellouze, N.: Speech Classification in Noisy Environment Using Subband Decomposition. In: ISSPA, vol. 1, pp. 409-412 (2003)

13. Mallat, S.: A Wavelet Tour of Signal Processing, 2nd edn. Academic Press, San Diego (1999)

14. Mallat, S., Hwang, W.L.: Singularity Detection and Processing with Wavelets. IEEE Trans. On Information Theory 38(2), 617-643 (1992)

15. Rosenfeld, A., Thurson, M.: Edge and Curve Detection for Visual Scene Analysis. IEEE Trans. Comput. 20, 562-569 (1971)

16. Sadler, B.M., Pham, T., Sadler, L.C.: Optimal and Wavelet-based Shock Wave Detection and Estimation. Journal of the Acoustical Society of America 104(2), 955-963 (1998)

17. Sadler, B.M., Swami, A.: Analysis of Multiscale Products for Step Detection and Estimation. IEEE Trans. Inform. Theory 45(3), 1043-1051 (1999)

18. Bouzid, A., Ellouze, N.: Open Quotient Measurements Based on Multiscale Product of Speech Signal Wavelet Transform. Research Letter in Signal Processing 2007, p. 5 (2007); Article ID 62521 doi:10.1155/2007/62521

19. Plante, F., Meyer, G.F., Ainsworth, W.A.: A Pitch Extraction Reference Database. In: Proc. Eurospeech 1995, pp. 837-840 (1995) 